Revista Arbitrada Interdisciplinaria KOINONIA

Año V. Vol V. N9. Enero - Junio 2020

Hecho el depósito de Ley: FA2016000010 ISSN: 2542-3088

FUNDACIÓN KOINONIA (F.K). Santa Ana de Coro. Venezuela.

Juan Carlos Erazo-Álvarez; Cecilia Ivonne Narváez-Zurita

http://dx.doi.org/10.35381/r.k.v5i9.662

\title{
Medición y gestión del capital intelectual en la industria del cuero - calzado en Ecuador
}

\section{Measurement and management of intellectual capital in the leather - footwear industry in Ecuador}

\author{
Juan Carlos Erazo-Álvarez \\ jcerazo@ucacue.edu.ec \\ Universidad Católica de Cuenca, Cuenca \\ Ecuador \\ https://orcid.org/0000-0001-6480-2270 \\ Cecilia Ivonne Narváez-Zurita \\ inarvaez@ucacue.edu.ec \\ Universidad Católica de Cuenca, Cuenca \\ Ecuador \\ https://orcid.org/0000-0002-7437-9880
}

Recibido: 15 de noviembre de 2019

Revisado: 30 de noviembre de 2019

Aprobado: 19 de diciembre de 2019

Publicado: 20 de enero de 2020

\section{RESUMEN}

En el Ecuador se busca un proceso de transformación productiva que permita avanzar hacia un mayor crecimiento y equidad, las cuales constituyen tareas que dependen del capital humano de los países y de la capacidad de las personas para resolver nuevos problemas mediante el ejercicio de conocimientos, competencias y habilidades adquiridas que elevan su productividad y su calidad de vida. En la industria de cuero y calzado de la provincia de Tungurahua se necesita elevar la efectividad organizacional de manera tal que contribuya al desarrollo de las estrategias en el cambio de la matriz productiva para lo que lo se plantea como objetivo la medición y gestión del capital intelectual del sector. La investigación es de tipo no experimental, de tipo mixta y de alcance descriptivo - explicativo. Los resultados más relevantes se presentan de acuerdo a la perspectiva de los clientes, empleados y dueños de los negocios.

Descriptores: Medición; gestión; capital intelectual; industria. (Palabras tomadas del Tesauro UNESCO). 
Revista Arbitrada Interdisciplinaria KOINONIA

Año V. Vol V. N9. Enero - Junio 2020

Hecho el depósito de Ley: FA2016000010

ISSN: 2542-3088

FUNDACIÓN KOINONIA (F.K). Santa Ana de Coro. Venezuela.

Juan Carlos Erazo-Álvarez; Cecilia Ivonne Narváez-Zurita

\begin{abstract}
In Ecuador, a process of productive transformation is sought to allow progressing towards greater growth and equity, which are tasks that depend on the human capital of the countries and on the ability of people to solve new problems through the exercise of knowledge, competences and acquired skills that increase their productivity and quality of life. In the leather and footwear industry of Tungurahua province, it is necessary to increase organizational effectiveness in a way that contributes to the development of strategies in the change of the productive matrix, for which the measurement and management of intellectual capital is set as an objective of the sector. The research is non-experimental, mixed and with descriptive - explanatory scope. The most relevant results are presented according to the perspective of customers, employees and business owners.
\end{abstract}

Descriptors: Measurement; management; Intellectual capital; industry. (Words taken from the UNESCO Thesaurus).

\title{
INTRODUCCIÓN
}

El capital intelectual resalta en el contexto de la gestión del conocimiento, entendiéndose éste como un conjunto de actividades realizadas con el fin de utilizar, compartir y desarrollar los conocimientos de una organización y de los individuos que en ella trabajan, encaminándolos a la mejor consecución de sus objetivos. En el Ecuador se busca un proceso de transformación productiva que permita avanzar hacia un mayor crecimiento y equidad, que son tareas que dependen del capital humano de los países, de la capacidad de las personas para resolver nuevos problemas mediante el ejercicio de conocimientos, competencias y habilidades adquiridas que elevan su productividad y su calidad de vida, siendo éstos parte de los 8 pilares fundamentales declarados en la agenda para la transformación productiva; cambio de la matriz productiva y talento humano.

La industria de cuero y calzado en el Ecuador está considerada como una de las actividades dinamizadoras de la economía, por lo que, en el plan nacional de desarrollo se prioriza entre otras a esta industria para el proceso de impulsar la transformación de la matriz productiva, que articulado con la agenda de transformación productiva 
establecido por el Ministerio de coordinación de la producción, empleo y competitividad (MCPEC) busca como eje de medición la productividad sistémica, que mide las capacidades productivas del país y la potencialidad de desarrollar los distintos sectores productivos, incluyendo indicadores de productividad, encadenamientos productivos y soberanía tecnológica(Erazo, 2018).

\section{DESARROLLO}

\section{La Gestión del capital intelectual}

La administración del capital intelectual es única en cada organización; se asume que el capital humano constituye la base para el logro del capital estructural de la empresa y la interacción del capital humano con el estructural permite la creación del capital relacional, en el centro de las tres formas de capital intelectual se encuentra el capital financiero o valor creado por la intersección de esos tres componentes (Valencia, 2005). La Gestión del Capital Intelectual se centra, desde un punto de vista estratégico, en la construcción y gestión de activos intelectuales, la renovación y actualización de los ya existentes, y el incremento del valor de todos ellos (Romero y Salvador, 2005).Según Monagas (2012) resulta generalmente aceptado considerar, dentro del señalado sistema de conceptos, que el proceso de gestión del "capital intelectual" puede asumirse como una actividad que tiene importancia significativa en la mejoría de los resultados de las organizaciones por la posibilidad que brinda para identificar elementos ocultos que puedan contribuir a dicho desarrollo.

Por lo que se han desarrollado modelos, metodologías y procedimientos de medición y gestión del capital intelectual que se encaminan a mejorar la gestión en las organizaciones.

Para el desarrollo de la presente investigación se analizan 13 metodologías de medición y gestión del capital intelectual, teniendo en cuenta los aspectos siguientes:

1. Posibilidades de adaptación a un sector

2. Elementos que apoyen la gestión del capital intelectual

3. Contribución a la satisfacción de los clientes, de sus empleados, de las entidades reguladoras, los donadores y otras organizaciones. 
Revista Arbitrada Interdisciplinaria KOINONIA

Año V. Vol V. N9. Enero - Junio 2020

Hecho el depósito de Ley: FA2016000010

ISSN: 2542-3088

FUNDACIÓN KOINONIA (F.K). Santa Ana de Coro. Venezuela.

Juan Carlos Erazo-Álvarez; Cecilia Ivonne Narváez-Zurita

4. Contribución a la capacidad de la organización para asegurar, administrar, controlar habilidades, recursos escasos y valiosos,

5. Contribución a la capacidad de la organización para ser innovadora, funcionar rápida y responsablemente.

6. Capacidad de la organización de convertir eficientemente las habilidades y recursos en bienes y servicios.

La fundamentación del análisis de estos aspectos, se relaciona con los elementos necesarios para lograr la efectividad organizacional (Azuara, 2018), (Cervera, 2011), (Montero, Leyva y Ballester, 2016),(Guerrero, 2013), en las pequeñas y medianas empresas agrupadas en un sector en correspondencia con el cambio de la matriz productiva establecido por el gobierno ecuatoriano.

\section{Metodologías de medición y gestión del capital intelectual}

1. Análisis de la gestión y la medición del capital intelectual en las empresas cubanas (Borras y Campos, 2013).

Tiene como principal objetivo evaluar la gestión del capital intelectual en empresas y detectar las variables que reciben menor atención, la gestión se mide a través de variables relacionadas con el capital humano, el estructural, el relacional, el social, se basa en una investigación anterior que tiene como objetivo identificar los principales factores intangibles que con mayor fuerza influyen en el desarrollo exitoso de las empresas cubanas. El resultado fue la identificación de 19 variables de capital intelectual.

Se aprecia la existencia de serias debilidades en la administración del capital intelectual y de bajos niveles de utilización de indicadores para su medición, resulta un diagnóstico muy importante, las variables utilizadas miden satisfacción de clientes, capacidad de innovación, entre otras.No se aprecian elementos que apoyen la gestión del capital intelectual 
2. Evolución de la medición y gestión del capital intelectual en una empresa del sector real en Colombia (Castellanos y Millán, 2010)

Se aplica una metodología en la empresa COL S.A, Colombia, que tiene como objetivo identificar, gestionar y medir el capital intelectual a través de la implementación de un sistema de gestión gerencial.

Se consideran diferentes etapas de evolución, se mide en primer lugar la eficiencia global de producción (EGP), la producción vendible y la gestión de la parte financiera, utiliza la base conceptual del modelo Skandia(Edvinsson, 2007) y el modelo de Cuadro de Mando Integral (Kaplan y Norton, 2000).

Los aspectos que contribuyen a la gestión del capital intelectual se centran en la aplicación de una tecnología de procesos, de información, cultura y estructura organizativa, la toma de acciones correctivas/preventivas, el análisis de desvío de metas, el tratamiento de anomalías, el cumplimiento del plan de estandarización y las auditorias.

Resulta útil para la gestión del capital intelectual ya que aporta elementos que identifican la capacidad de la organización de convertir eficientemente las habilidades y recursos en bienes y servicios.

No se profundiza en la contribución a la capacidad de la organización para asegurar, administrar, controlar habilidades, recursos escasos y valiosos, elementos de la efectividad organizacional.

3. Gestión del Conocimiento y del Capital Intelectual en una PYME delsector textil (Romero y Salvador, 2008)

Se miden los indicadores del cuadro de mando Integral (Kaplan \& Norton, 2000), se elaboran estrategias para gestionar el capital intelectual relacionadas con: mejorar la formación para aumentar las capacidades de los empleados, facilitar la rotación, disminuir los costos de mantenimiento, mejorar la flexibilidad, y el tiempo de respuesta. 
Revista Arbitrada Interdisciplinaria KOINONIA

Año V. Vol V. N9. Enero - Junio 2020

Hecho el depósito de Ley: FA2016000010

ISSN: 2542-3088

FUNDACIÓN KOINONIA (F.K). Santa Ana de Coro. Venezuela.

Juan Carlos Erazo-Álvarez; Cecilia Ivonne Narváez-Zurita

Se crean grupos para compartir información y conocimiento, adscritos al organigrama con documentación propia de la empresa y de sus procesos, para facilitar la transmisión y el registro del conocimiento interdepartamental, las actuaciones en mantenimiento y la producción. No se profundiza en elementos para contribuir a la gestión del capital intelectual.

4. Metodología para la gestión del capital intelectual en las pequeñas y medianas empresas en México (Morales, Modelo de gestión de capital intelectual para la pequeña y mediana empresa en México, 2013)

Se consideran tres etapas: la primera es la medición, con el propósito de medir los indicadores en tres bloques, estructura interna, operatividad de la empresa y flujos de información; capital humano: conocimiento, propiedad de quien los posee; capital relacional, con interacción con el exterior, imagen, relaciones.

La segunda etapa es la selección donde se define cual es el conocimiento costeable y conveniente para registrar en las diversas modalidades de propiedad intelectual.

La tercera etapa es explotación y gestión, que se considera que se realice por medio de las tecnologías de la información, de las ventas y las franquicias.

Se dedica a profundizar en la propiedad intelectual, no se observan elementos relacionados con la contribución a la capacidad de la organización para asegurar, administrar, controlar habilidades, recursos escasos y valiosos, para ser innovadora, funcionar rápida y responsablemente y para convertir eficientemente las habilidades y recursos en bienes y servicios, elementos de la efectividad organizacional.

5. Capital intelectual en la gestión pública: caso del método intellectus (Harms, Avila, Rados y Rodríguez, 2015)

Tiene como objetivo comprobar la cohesión conceptual entre capital intelectual y la gestión pública, se aplica el modelo intellectus, se considera como capital acelerador al capital de emprendimiento e innovación que incluye resultados de la innovación, emprendimiento e innovación, esfuerzo en innovación, actitudes y capacidad de 
Revista Arbitrada Interdisciplinaria KOINONIA

Año V. Vol V. N9. Enero - Junio 2020

Hecho el depósito de Ley: FA2016000010

ISSN: 2542-3088

FUNDACIÓN KOINONIA (F.K). Santa Ana de Coro. Venezuela.

Juan Carlos Erazo-Álvarez; Cecilia Ivonne Narváez-Zurita

emprendimiento. No se aprecian elementos que apoyen la gestión del capital intelectual, se enfoca a la medición y a la relación con la gestión pública.

6. Capital intelectual en mercados en desarrollo. "El caso Paraguay" (Sosa, 2013)

La metodología tiene como propósito valorar la importancia de los activos intangibles en el desempeño de las empresas paraguayas, el desempeño se mide utilizando informes financieros y no financieros con la adaptación del modelo de Kolhi y Jaworski(Kohli y Jaworski, 1990). Se considera que el capital intelectual es uno de los constructos de mayores proyecciones dentro de los activos intangibles, aunque no es sinónimo de activos intangibles, lo divide en tres categorías, capital interno(estructural), capital externo (relacionado con el cliente) y capital humano.

Se pretende la construcción de una metodología que se adapte a países emergentes, los indicadores se construyen sobre trabajos empíricos consultados y adaptados al sector industrial.La necesidad de medir el desempeño hace que se incluyan resultados financieros, de mercado y de innovación como, calidad de los productos, satisfacción de los clientes, nuevos productos/servicios, volumen de ventas, utilización presupuesto de publicidad, imagen y reconocimiento de la marca.Resulta de utilidad para las organizaciones por la integración de resultados financieros, de mercado y de innovación, lo que influye en la efectividad organizacional, no obstante, se enfoca al diagnóstico y a la medición más que a la gestión.

7. Metodología de medición de impacto del capital intelectual en la innovación en empresas: una perspectiva de México(Montejano \& Citlalli, 2013)

Esta investigación analiza el valor y uso del capital intelectual y su relación con la innovación en las organizaciones, se aplica a 149 empresarios, en la ciudad de Aguascalientes, México.Se expone que existe una relación directa entre el grado de innovación de la empresa y su capacidad para ampliar el capital intelectual, innovación en estrategias de marketing, en marcas, en la búsqueda de elementos que puedan ser objeto de contratos de licencias, en investigación y desarrollo, en el uso y diseño de 
infraestructura y en la forma en la que se estimula y fomenta el desarrollo personal y profesional de los empleados para provecho de la empresa.Su utilidad radica en la integración que logra entre el capital intelectual y la innovación, no obstante, no se observa con claridad la contribución a la capacidad de la organización para asegurar, administrar, controlar habilidades recursos escasos y valiosos.

8. Capital intelectual. Una propuesta para clasificarlo y medirlo (Alama, Martin de Castro y López, 2006)

Esta metodología de medición y clasificación identifica indicadores utilizados por los autores de los trabajos empíricos revisados y selecciona en correspondencia a la frecuencia de uso.Se elige un total de 47 indicadores para la medición del capital intelectual, para medir el capital humano se consideraron indicadores que miden el nivel de conocimiento y habilidad de los empleados, así como su creatividad y experiencia para el desarrollo eficaz de sus tareas. Resulta muy interesante por el amplio trabajo de consulta bibliográfica que realiza y la sistematización de indicadores más utilizados en diferentes modelos, metodologías y procedimientos, se centra en la clasificación y medición, no se aprecian elementos relacionados con la gestión del capital intelectual y la efectividad organizacional.

9. Indicadores de capital intelectual y su relación con el rendimiento. Un análisis empírico (Ochoa, Prieto, \& Santidrian, 2013)

En la metodología se relacionan los indicadores de capital humano y de capital estructural y los resultados económico-financieros de las cuentas anuales de 211 empresas españolas, pertenecientes a todos los sectores de actividad -excepto instituciones financieras y sector primario-, con más de 25 trabajadores, se calcula el valor agregado correspondiente a la suma de la eficiencia del capital humano y estructural y su vinculación con medidas de creación de valor.

Se insiste que el interés del estudio del capital intelectual, del capital humano y estructural, se debe a la supuesta capacidad del mismo para alentar la creación de 
valor, por lo que se analizan variables relacionadas con crecimiento en ventas, el tamaño de la empresa, la relación entre la deuda total y el total de fondos propios, la antigüedad de la empresa y el sector al que pertenece.

Su importancia radica en la valoración de que se realiza del aporte del capital intelectual a la creación de valor en las organizaciones lo que contribuye a acumular experiencias para su gestión.No se aprecian elementos relacionados con la capacidad de la organización de convertir eficientemente las habilidades y recursos en bienes y servicios.

10. Revelación del capital intelectual en empresas cotizantes del mercado de capitales argentino (Ficco, Analía y Sader, 2013)

Se realiza una metodología para el estudio de las prácticas de revelación de información sobre intangibles adoptadas por las empresas que cotizan en el mercado de valores de Buenos Aires, con el objeto de identificar, la información que presentan sobre activos intangibles en los estados de publicación y determinar qué tipo de información revelan voluntariamente en la memoria y en la información complementaria sobre su capital intelectual.

Se valora, el tamaño de la empresa y su crecimiento, su rentabilidad, el nivel de endeudamiento, la rentabilidad sobre ventas, la rotación del activo, el ratio de endeudamiento y la cantidad de activos intangibles reconocidos a través del ratio entre activos intangibles y activo total. Se define un índice de revelación voluntaria de información sobre capital intelectual que se fundamenta en los elementos de información no obligatoria que revelan las empresas sujetas a estudio, los cuales se obtuvieron a partir del análisis de la memoria y de la información complementaria a los estados financieros.

Los elementos fueron agrupados en las tres categorías básicas en que habitualmente se agrupan los componentes del capital intelectual.Esta metodología se relaciona con la intención de integrar en la contabilidad los elementos del capital intelectual, mediante la 
Revista Arbitrada Interdisciplinaria KOINONIA

Año V. Vol V. N9. Enero - Junio 2020

Hecho el depósito de Ley: FA2016000010

ISSN: 2542-3088

FUNDACIÓN KOINONIA (F.K). Santa Ana de Coro. Venezuela.

Juan Carlos Erazo-Álvarez; Cecilia Ivonne Narváez-Zurita

información voluntaria de las empresas, no se observan elementos relacionados con la gestión del capital intelectual.

11. El papel del capital intelectual en la innovación tecnológica. Una aplicación a las empresas de servicios profesionales de España (Martin de Castro, Alama, Navas y López, 2009)

El objetivo específico de esta metodología es determinar la influencia del capital humano, estructural y del capital relacional de la empresa, en la capacidad de la misma para innovar en productos/proyectos y servicios, se aplica a una muestra de empresas españolas de servicios profesionales.En la etapa I se realiza un análisis factorial exploratorio para poder determinar los componentes básicos del capital intelectual, después se aplica un modelo de regresión lineal múltiple para contrastar la influencia de los distintos tipos de capital intelectual sobre la capacidad de innovación.

Se comprueba que existe una relación positiva y estadísticamente significativa únicamente con la dimensión del capital humano relativo a la experiencia y habilidades de los trabajadores, en el capital estructural con la cultura de la organización y las competencias en tecnologías de la información y en el capital relacional con las relaciones con los clientes y las alianzas y reputación empresarial.

Sobre el efecto de los tres capitales en la innovación se comprueba relación positiva, fuerte y estadísticamente significativa de las relaciones con los clientes, las alianzas, la reputación y las competencias de la empresa en tecnologías de la información.Esta metodología se enfoca en la medición de impacto del capital intelectual en la innovación, con cierta similitud con la realizada por (Montejano y Citlalli, 2013).No se observan elementos de la gestión del capital intelectual.

12. Capital social, capital intelectual e innovación de producto. Evidencia empírica en sectores manufactureros intensivos en tecnología (Delgado, Martin de Castro, Navas y Cruz, 2013) 
Se propone una metodología, específicamente para el capital social, como componente del capital intelectual y se determina su influencia sobre la innovación de producto. Los resultados muestran que la relación entre cada una de las dimensiones de capital social, de manera independiente, y la innovación de producto es positiva y altamente significativa. La visión compartida, es la dimensión que mayor poder explicativo tiene sobre la innovación de producto, a continuación, aparece la dimensión red social, lo que indica que para lograr una innovación de producto el intercambio de ideas y las discusiones constructivas adquieren un papel relevante. Ello se debe a que el trabajo en grupo desarrolla nuevas ideas que no surgen de manera individual y proporciona una mayor amplitud de conocimientos.

En último lugar, se encuentra la confianza y apoyo social, de manera que los datos parecen indicar que, aunque no tienen tanta importancia como los otros dos factores de capital social, la ayuda mutua proporcionada por los compañeros de trabajo supone un aspecto clave a la hora de conseguir una innovación de producto.Respecto a las variables de control, sólo la antigüedad de la empresa, y concretamente dentro del modelo donde se incluyen las tres dimensiones de capital social, tiene una leve influencia significativa y positiva.

De modo que aquellas organizaciones que lleven más tiempo en el mercado tendrán unas relaciones personales e informales más sólidas que aquellas empresas más jóvenes.Resulta muy interesante esta metodología ya que profundiza en la medición de elementos menos investigados como intercambio informal de ideas e información, visión compartida, entusiasmo con sus objetivos, que pueden resultar útiles para la gestión del capital intelectual.

No obstante, no se observan elementos relacionados con la capacidad de la organización de convertir eficientemente las habilidades y recursos en bienes y servicios y la capacidad para asegurar, administrar, controlar habilidades, recursos escasos y valiosos. 
13. Gestión del Capital Intelectual: Un reto para la gerencia de la pequeña y mediana empresa latinoamericana. (Romero, Pascual y Hernández, 2012)

La metodología tiene como objetivo determinar el nivel de madurez de las capacidades para gestionar el capital intelectual en la pequeña y mediana empresa y su relación con el desarrollo alcanzado por las capacidades de la gestión del conocimiento organizacional, como consecuencia de la magnitud de la influencia que sobre ellas ejercen en conjunto, las tecnologías de la información, la cultura organizacional y las capacidades del capital humano.

Se diseña una guía de comparación que contiene las áreas y parámetros que caracterizan los niveles de madurez de las capacidades que debe poseer una empresa para gestionar su capital intelectual, Dicha caracterización toma como base el tipo o magnitud de la relación entre las variables del estudio para definir los diferentes estadios, los cuales representan diferentes contextos de madurez, desde el más sencillo hasta el más complejo.

Se demuestra que las pymes latinoamericanas reportan altos niveles de madurez en sus capacidades para gestionar el capital intelectual, se comprueba que estos niveles de madurez no fueron alcanzados como consecuencia de la influencia, que sobre las capacidades para gestionar conocimiento organizacional han podido ejercer en conjunto, las tecnologías de la información, la cultura organizacional y las capacidades del capital humano.

Esta metodología contiene elementos que resultan útiles para gestionar el capital intelectual, con sugerencias muy interesantes investigadas y fundamentadas relacionadas con las posibilidades y niveles de madurez de las empresas para la mencionada gestión del capital intelectual.Se basa fundamentalmente en la relación con la gestión del conocimiento, elemento clave para el desarrollo del capital intelectual, no relaciona la investigación realizada con la efectividad organizacional.

Se aprecian que de las 13 metodologías estudiadas, 6 de ellas, aportan elementos para la gestión del capital intelectual (Castellanos y Millán, 2010), (Sosa, 2013), (Montejano y Citlalli, 2013), (Ochoa, Prieto y Santidrian, 2013), (Delgado, Martin de Castro, Navas y 
Cruz, 2013), (Romero, Pascual y Hernández, 2012), otras profundizan en la gestión del conocimiento y la propiedad intelectual (Romero y Salvador, 2008), (Morales, Modelo de gestión de capital intelectual para la pequeña y mediana empresa en México, 2013), (Romero, Pascual y Hernández, 2012).

Se observan que (Sosa, 2013), (Alama, Martin de Castro y López, 2006) se enfocan al diagnóstico y a la medición más que a la gestión, así como a la sistematización de indicadores más utilizados en diferentes modelos, metodologías y procedimientos.

Resulta interesante la medición de impacto del capital intelectual en la innovación que realizan (Martin de Castro, Alama, Navas y López, 2009) y (Montejano y Citlalli, 2013), la intención de integrar en la contabilidad los elementos del capital intelectual (Ficco, Analía y Sader, 2013) y la relación con la gestión pública (Harms, Avila, Rados y Rodríguez, 2015).

No se observa en las metodologías estudiadas el enfoque de sector, así como la contribución a la efectividad organizacional declarada explícitamente, no obstante, se destaca que en todas se identifica la relación del capital intelectual con la satisfacción de los clientes, de los empleados, de las entidades reguladoras, los donadores y otras organizaciones, elementos definidos por (Montero, Leyva y Ballester, 2016),(Guerrero, 2013), (Cervera, 2011) de la mencionada efectividad organizacional.

No se profundiza en otros elementos de la efectividad organizacional (Azuara, 2018), (Cervera, 2011) como la contribución a la capacidad de la organización para asegurar, administrar, controlar habilidades recursos escasos y valiosos en (Castellanos y Millán, 2010), (Morales, Modelo de gestión de capital intelectual para la pequeña y mediana empresa en México, 2013), (Montejano y Citlalli, 2013), (Delgado, Martin de Castro, Navas, y Cruz, 2013).

Otro elemento de la efectividad organizacional (Azuara, 2018), (Cervera, 2011) como la capacidad de la organización de convertir eficientemente las habilidades y recursos en bienes y servicios no se aprecia en (Delgado, Martin de Castro, Navas y Cruz, 2013), (Ochoa, Prieto y Santidrian, 2013), (Morales, Modelo de gestión de capital intelectual para la pequeña y mediana empresa en México, 2013). 
Como resumen de la bibliografía valorada en el capítulo se puede sistematizar que resulta necesario que los modelos, metodologías y procedimientos para la medición y gestión del capital intelectual se adapten a las exigencias del entorno institucional en que se desarrollan las organizaciones, con el objetivo de que resulten efectivos y contribuyan a la mejora de la gestión.La importancia de establecer mecanismos para la incorporación de las micro, pequeñas y medianas unidades productivas y de servicios en el desarrollo de sus capacidades para explotar el conocimiento en la búsqueda de la efectividad organizacional, por lo que resulta interesante el estudiode las potencialidades para la gestión del capital intelectual en las mismas.

Las metodologías para la gestión del capital intelectual estudiadas integran elementos muy útiles para la presente investigación y se identifican aspectos aun no profundizados, que pueden resultar novedosos como la gestión del capital intelectual, su contribución a la mejora de la efectividad organizacional en pequeñas y medianas empresas, en correspondencia con el cambio de la matriz productiva establecido por el gobierno ecuatoriano.

\section{METODOLOGÍA}

De acuerdo a las contribuciones de este estudio, la investigación fue de tipo no experimental pues no se manipularon variables sino se estudiaron los fenómenos del sector en su estado actual para posteriormente analizarlos y explicarlos. La investigación tuvo un alcance descriptivo - explicativo pues las variables planteadas fueron descritas y explicadas en el presente documento. Con respecto a la finalidad, se definió que esta investigación fue transversal pues la recolección de los datos se realizó en un solo momento y se explicó sus relaciones e incidencias.

Para esta investigación se utilizó el método histórico - lógico con el fin desarrollar el marco teórico y el estado de situación de la investigación realizada. Además, se utilizó método analítico - sintético pues este separa el objeto del estudio en dos partes: el análisis, encargado de manejar los juicios, y por otro lado la síntesis que considera los objetos como un todo. Así mismo, se utilizó el método deductivo - inductivo pues con el 
primero se organizaron los hechos conocidos y se han extraído conclusiones mientras que con el segundo se establecieron conclusiones generales basándose en los hechos recopilados.

Con respecto a las técnicas, se utilizaron encuestas con el fin de levantar toda la información posible para poder analizar las variables. Por este motivo, el instrumento utilizado fue el cuestionario estructurado (Bernal, Erazo y Narváez, 2019).Se aplicódicho cuestionario a los clientes de los negocios de producción de cuero y calzado de la Provincia de Tungurahua, con el objetivo de medir la satisfacción de los mismos con los productos ofertados, se hace una prueba piloto a 94 clientes y se perfeccionan las preguntas en correspondencia con las sugerencias recibidas. Se seleccionó la muestra teniendo en cuenta la fórmula para extrapolar a poblaciones que se desconoce:

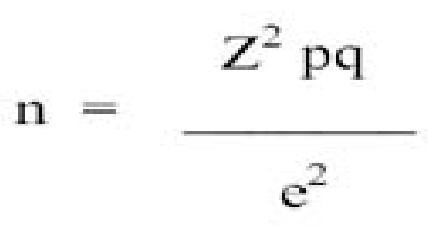

Donde:

$\mathrm{z}=$ Valor de $\mathrm{z}$ correspondiente al nivel de confianza;

Un nivel de confianza del 95\% (también se expresa: $\alpha=.05$ )

corresponde a $z=1.96$ sigmas o errores típicos; $z=2$ (dos sigmas) corresponde a un 95.5\% (aproximadamente, $\alpha=.045$ ).

$\mathrm{pq}=$ Varianza de la población

Como la varianza de la población no se conoce, se coloca la varianza mayor posible porque a mayor varianza hará falta una muestra mayor.

$p=$ proporción de respuestas en una categoría (síes, respuestas correctas, unos en la codificación usual, etc.)

$q$ = proporción de repuestas en la otra categoría (noes, ceros en la codificación usual).

La varianza en los ítems dicotómicos (dos respuestas que se excluyen mutuamente) es igual a pq y la varianza mayor (la mayor diversidad de respuestas) se da cuando $p=q=$ 
.50 (la mitad de los sujetos responde sí y la otra mitad responde no) por lo que en esta fórmula [1] pq es siempre igual a (.50) (.50) $=.25$ (es una constante).

$\mathrm{e}=$ Error muestral

Se representa con la letra e (no es el único símbolo que se utiliza) que significa error o desviación posible cuando se extrapolan los resultados. Es el margen de error que se acepta. Si el margen máximo de error es $10 \%$, en la fórmula se pone e $=0.10$

El tamaño de la muestra (cociente o resultado de la fórmula) será mayor según sea mayor el nivel de confianza y la varianza esperada en la población (numerador en la fórmula) y según sea menor el margen de error que estamos dispuestos a admitir (denominador en la fórmula) (Morales, 2017, p. 5-6).Los resultados del cálculo de la muestra son los siguientes:

CONCEPTO

Z: coeficiente de

confianza

p: proporción que "si"

q: proporción que "no"

e: error máximo

permitido

Tamaño de la muestra

\section{VALORES}

1.96

0.50

0.50

0.10

94

Se establece una muestra de 94.

Se aplicótambién un cuestionario a empleados o trabajadores, con el objetivo de valorar su nivel de satisfacción con el trabajo que realizan, así como el apoyo tecnológico, la capacidad de información, la gerencia en los procesos,el desarrollo del trabajo en equipo, y las iniciativas, elementos que miden el grado de madurez del capital intelectual (Romero, Pascual y Hernández, 2012), se aplica a muestra piloto y se reajustan las preguntas con dificultades para su comprensión. 
Se selecciona una muestra de 94.

Se realizóotro cuestionario, con el objetivo de explorar la percepción de los dueños de los negocios sobre la capacidad de convertir eficientemente las habilidades y recursos en bienes y servicios y para asegurar, administrar, controlar habilidades y recursos escasos y valiosos, elementos que miden la efectividad organizacional. (Azuara, 2018), (Cervera, 2011)

En el cuestionario se explora sobre el comportamiento humano-individual, apoyo tecnológico, capacidad de información, gerencia en los procesos, desarrollo del trabajo en equipo y mejoras por iniciativa propia, elementos del grado de madurez del capital intelectual (Romero, Pascual y Hernández, 2012).

Se aplica a 30 dueños de negocios.

\section{RESULTADOS}

En la medición de la efectividad de los negocios, los resultados muestran que más del $50 \%$ de los clientes no conocen los zapatos de lujo que se producen, si consumen los de corte casual, formal, botas e industrial. La frecuencia de compra de los productos es cada 4 meses aproximadamente.
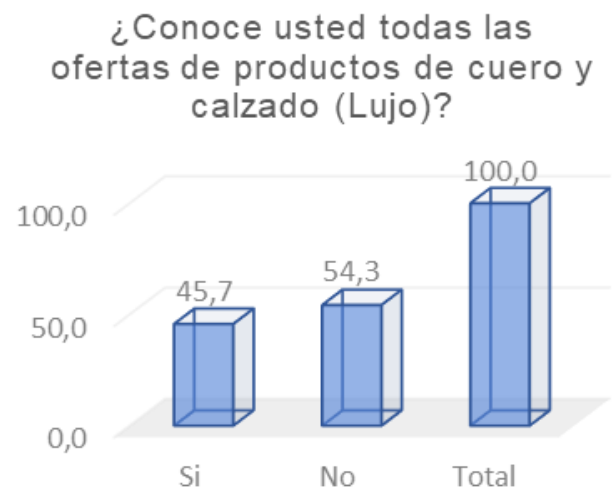

¿Con qué frecuencia compra

productos de cuero y calzado?

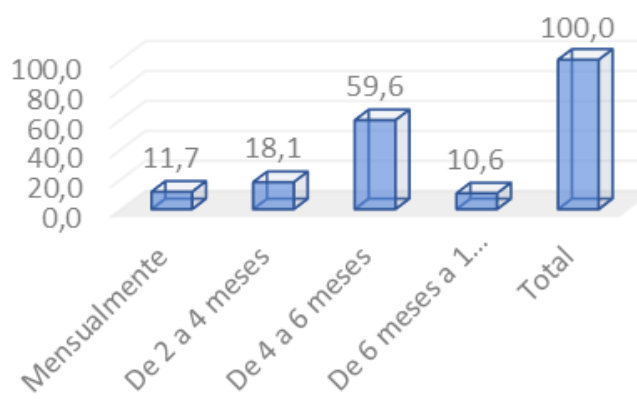

Un resultado muy importante para los negocios es que el $100 \%$ de los clientes encuestados consideran los productos muy útiles y útiles, sin embargo, al calificar la 
rapidez del servicio la media está en 2,76 , lo que evalúa el servicio como adecuado, si bien no es un efecto negativo, se identifican posibles áreas de mejora para lograr la apreciación de rápido o muy rápido, elementos que pueden contribuir a la ventaja competitiva.

¿Es de utilidad el uso de los productos de cuero y calzado para usted?

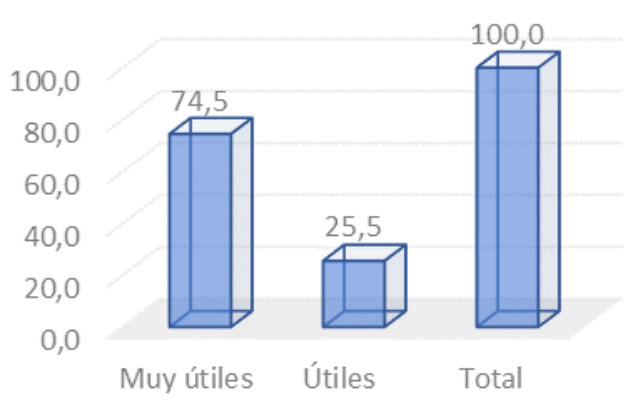

¿Cómo califica la rapidez en el servicio prestado?

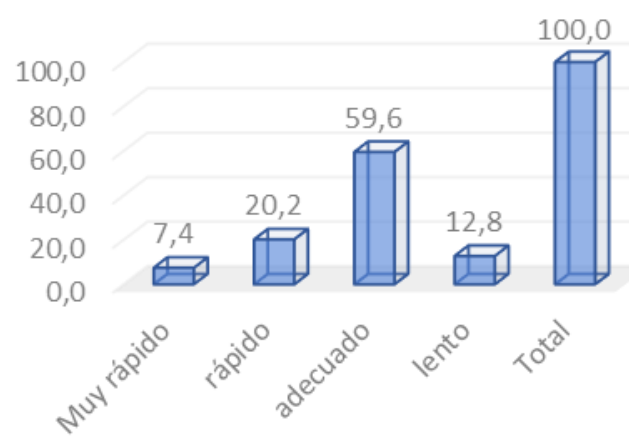

Con respecto a las condiciones de los negocios para brindar el servicio, más del $60 \%$ de los clienteslo califican como regulares y malos, si bien en ocasiones se necesita apoyo financiero para realizar inversiones, se puede recurrir además a explorar las iniciativas propias de los empleados en función de mejorar las mencionadas condiciones.

La pertinencia de la información que brinda el negocio sobre sus productos, es percibida como poco pertinente (media 3,5), debe entenderse que las tecnologías de información y las telecomunicaciones son un medio, en la actualidad, para transmitir y gestionar datos, información y conocimiento y este es un factor fundamental para la creación de riquezas.

Los clientes consideran que estos negocios no son muy profesionales (media 3,22), o sea, no perciben que los empleados desarrollan notoriamente el servicio que deben prestar, o no poseen las competencias técnicas y personales necesarias para el desarrollo de su profesión. 
La calidad de los productos es valorada de buena (media 2,83), aunque se considera un poco peor y muy por debajo de la competencia (media 4,31), no se aprecia que estos negocios sean capaces de proporcionar productos y servicios con mayor eficacia y eficiencia frente a sus competidores. Además de tener productos con precios menos aceptables que la competencia (media 3,98).

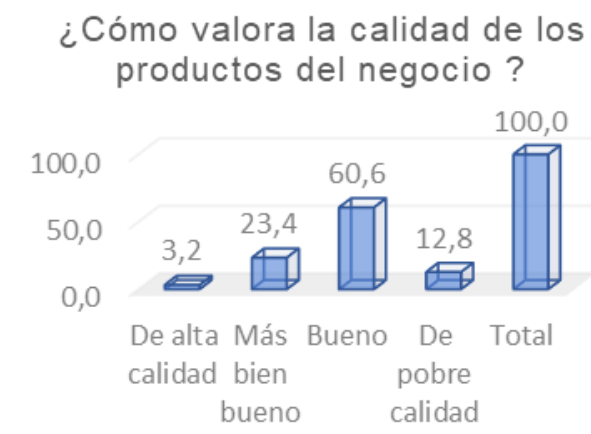

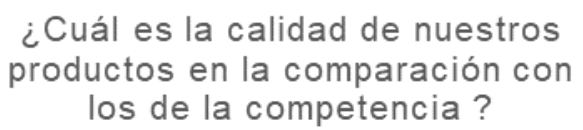

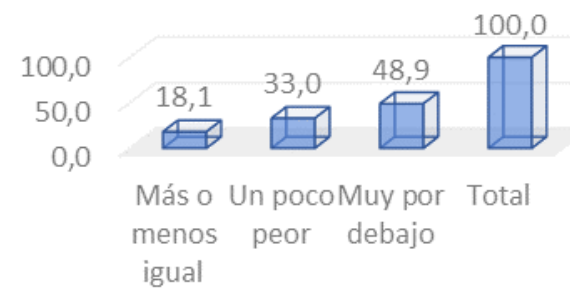

Los clientes califican el trato que reciben de los empleados de regular a malo (media 4,24), ser amable, es tener actitud complaciente y afectuosa, la amabilidad es una cualidad en la que brillan otras cualidades o rasgos, que casi siempre hay que entrenar, hay un punto de comprensión, de empatía, hay que enfatizar en la importancia de la cortesía y el trato amable como la mejor manera de atender a los clientes.Esto trae como resultado, que los clientes consideren no probable recomendar los productos que ofertan estos negocios a los demás (media 3,86 ).

Como se muestra al medir en estas pequeñas empresas la satisfacción de los clientes, los resultados apuntan que, se consideran los productos muy útiles y con buena calidad, no obstante, prevalecen condiciones regulares para ofrecer el servicio, la información que se brinda es poco pertinente, no se aprecia profesionalidad en los negocios, el trato de los empleados es de regular a malo.

Todo lo anterior hace que se perciba que los productos tienen menos calidad y precios menos aceptables que la competencia y es poco probable su recomendación a otros. 
Otro elemento para medir la efectividad se relaciona con la incidencia que en los negocios tienen las entidades reguladoras.

El gobierno considera que hay que lograr la integración del sector público, privado y la academia para captar inversiones, incrementar productividad y generar empleo y que la política económica debe adaptarse a las necesidades del sector Se logra el incremento de la economía mediante la utilización de créditos, en algunos casos, no reembolsables con aquellas organizaciones que permitan desarrollarlas.

Se analiza la efectividad de las empresas, teniendo en cuenta además la satisfacción de los trabajadores, los resultados muestran que solo un $3 \%$ tiene definidas sus funciones y responsabilidades en el negocio que trabaja, es muy importante que el trabajador tenga claridad en lo que debe hacer, esto puede afectar la motivación por el trabajo, la satisfacción al cliente y los resultados del negocio.

Más del 70 \%de los trabajadores no conoce los riesgos y las medidas de prevención relacionadas con su puesto de trabajo, resulta indispensable evitar que los trabajadores sufran daños derivados del trabajo, ya sean estos accidentes, enfermedades, patologías o lesiones y la herramienta fundamental es la prevención.

Esto da como resultado que el 53 \%de los trabajadores valoren como insegura su área de trabajo y se identifica como una de las causas fundamentales que el $60 \%$, expresen que no se les facilita los medios de protección individual necesarios para su trabajo.

La suma de dinero que recibe de forma periódica un trabajador de su empleador por un tiempo de trabajo determinado o por la realización de una tarea específica o fabricación de un producto determinado aún resulta la motivación fundamental en el desarrollo de su trabajo, en las empresas investigadas el $66,7 \%$ de los empleados consideran malas las condiciones salariales.

El desarrollo profesional hace que un trabajador llegue a un nivel óptimo de conocimientos necesarios para desempeñarse en el área de su interés, teniendo además la influencia, de cualidades personales como la actitud, insistencia, la proactividad y la propia lucha por mejorar, el $100 \%$ de los trabajadores califican de regulares a malas las oportunidades que les brindan para su desarrollo profesional. 
Revista Arbitrada Interdisciplinaria KOINONIA

Año V. Vol V. N9. Enero - Junio 2020

Hecho el depósito de Ley: FA2016000010

ISSN: 2542-3088

FUNDACIÓN KOINONIA (F.K). Santa Ana de Coro. Venezuela.

Juan Carlos Erazo-Álvarez; Cecilia Ivonne Narváez-Zurita

¿Cómo usted valora las condiciones salariales?

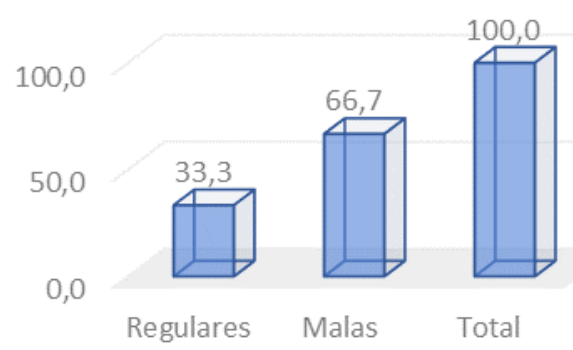

¿Como califica las oportunidades que les brindan para su desarrollo profesional?

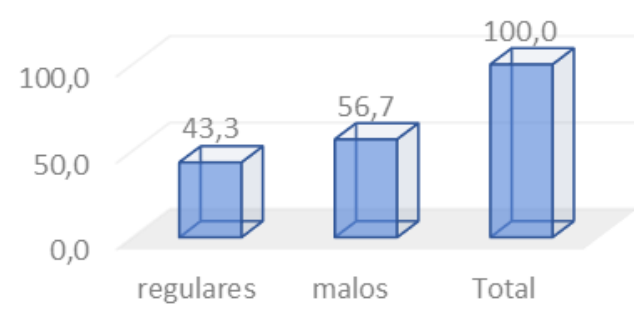

El aprendizaje organizacional considera que las empresas del futuro ya no podrán restringirse sólo a la calificar a sus trabajadores, sino más bien a organizar el trabajo de tal modo que toda la organización tenga las oportunidades de aprender, aunque resulte contradictorio el resultado de que el $86.7 \%$ de los trabajadores estén satisfechos con la formación específica recibida, al margen de la establecida en el plan, se advierte un área de mejora relacionada con la gestión del capital intelectual que logre que los trabajadores valoren oportunidades para el desarrollo profesional.

Una buena comunicación es básica para que los trabajadores se sensibilicen ante la cultura empresarial, es importante que los empleados se comprometan con la organización, más allá de las compensaciones económicas, el ambiente de trabajo de cordialidad y confianza induce a estar más concentrados en las actividades que real iza, es un resultado alentador el hecho de que el $86,7 \%$ se sienta involucrado con los éxitos y fracasos de su área de trabajo, además que el $53 \%$ conozca las tareas que desempeñan otras áreas del negocio.

El $70 \%$ de los trabajadores, califica de regular la información que recibe sobre el desempeño de su trabajo, esta retroalimentación permite mejorar la productividad, poder establecer políticas de compensación, mejorar las políticas de ascenso, detectar errores en el diseño del puesto del empleado y establecer planes de formación.

El $40 \%$ de los trabajadores expresan no estar motivados con el trabajo que realizan y un $56 \%$ algo motivados, sentirse motivado significa identificarse con el objetivo a 
cumplir y, por el contrario, sentirse desmotivado representa la pérdida de interés y de significado de la meta a alcanzar y puede provocar la imposibilidad de conseguirlo.

El concepto de efectividad surge de los postulados de la eficiencia y la eficacia, pero hace énfasis en la necesaria satisfacción del cliente, mediante el desarrollo de las capacidades intelectuales del capital humano, en función del cumplimiento de las metas.

¿Cómo califica la información

que recibe del desempeño de su trabajo?

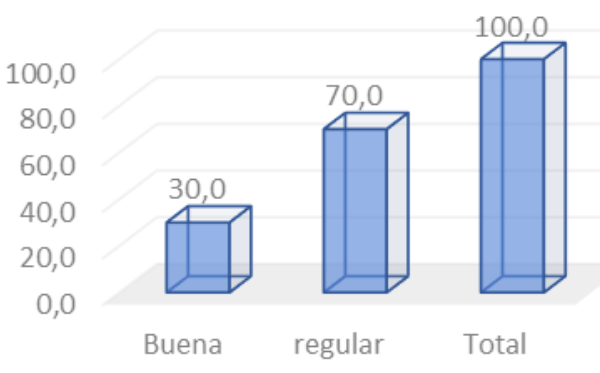

¿Está motivado con el trabajo que desarrolla?

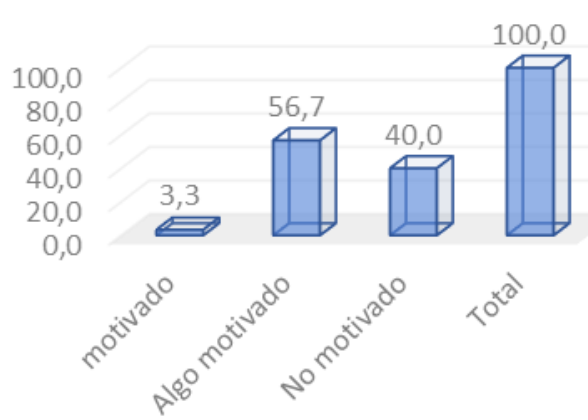

Se integran al cuestionario aplicado a los trabajadores, elementos que indagan sobre este nivel de madurez, los resultados muestran que más del $50 \%$ de los trabajadores, consideran desorganizado el trabajo en el área en que laboran.

Es importante desterrar del negocio la clásica organización funcional y transitar a la gestión por procesos, que aporta una visión y herramientas con las que se puede mejorar y rediseñar el flujo de trabajo para hacerlo más eficiente y adaptado a las necesidades de los clientes.

Este resultado se corresponde con que más del $70 \%$ de los trabajadores consideren regularmente y mal repartidas las cargas de trabajo entre ellos.

La reorganización del trabajo se aplica a la naturaleza del trabajo, implica suprimir las actividades no esenciales que se encuentran más allá de la capacidad de una determinada persona, se requiere un análisis detallado para identificar las demandas y capacidades mentales, físicas y sensoriales involucradas en la actividad. 
La aplicación de la gestión por procesos contribuye a mejorar la repartición de las tareas, ya que supone la integración del conjunto de las labores que se demandan, los recursos y tiempo necesarios, la asignación de trabajos a los nodos de proceso correspondientes, lo que conlleva a la mejora en el uso de los recursos y el rendimiento del sistema, en función de la satisfacción del cliente.

La tecnología ha reducido las barreras para realizar negocios, incrementar ingresos, mejorar procesos e implementar nuevas herramientas en las empresas, es una necesidad fundamental que permite insertarse en el mercado, más del $60 \%$ de los trabajadores evalúa de regular a malo, el apoyo tecnológico para realizar su trabajo. La empresa que no está preparada para que sus trabajadores hagan propuestas de mejoras en el trabajo, se retrasa en la innovación como un elemento adicional de mejora, se aprecia que más del $60 \%$ de los trabajadores, consideran algo posible e imposible proponer mejoras en su trabajo, por lo que se observa que no hay una gestión eficiente que haga posible la participación de los empleados en la administración.

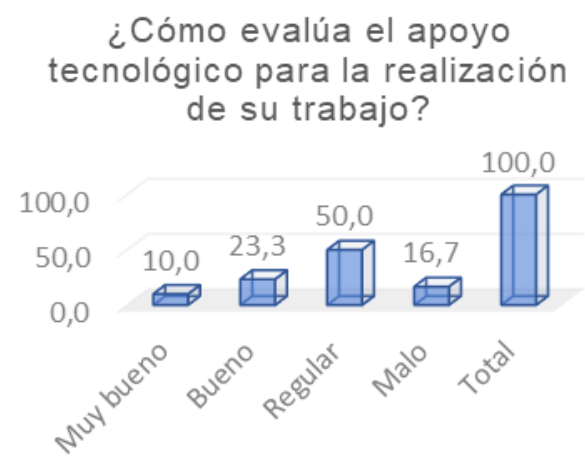

\section{¿Es posible proponer mejoras en su trabajo?}

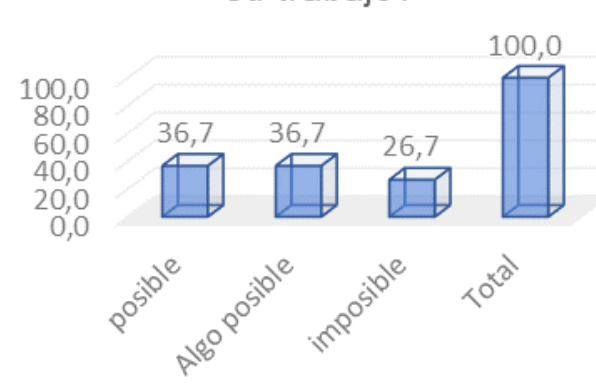

La creatividad está presente en todos los ámbitos de la vida, cuando somos creativos en el negocio las propuestas y soluciones adquieren un nivel de responsabilidad y de seriedad, es responsabilidad de la gerencia impulsar su en el trabajador, los resultados apuntan que el $73 \%$ califican de regular y mala, la contribución de los empleados a encontrar soluciones creativas. 
Es importante la capacidad que tiene una empresa para generar ideas o alternativas de gestión y materializarlas en productos, con la introducción de cambios en los procesos para optimizar el servicio o producto, se evidencian reservas para estas empresas, ya que más del $60 \%$, de los trabajadores califica de regular a mala la voluntad de los directivos de innovar para mejorar la calidad de los productos.

No se observan además intenciones de promover la innovación para mejorar la calidad, pues el $80 \%$, de los trabajadores no ha participado en eventos e intercambios de calidad e innovación.

Se aplica el cuestionario a 30 dueños de negocio, 26 hombres, el 56 \% tiene educación secundaria y solo un $3 \%$ estudios de posgrado.

El $100 \%$ de los negocios cuenta con registro único de contribuyentes (RCC) o registro impositivo simplificado ecuatoriano (RISE), el 83 \% lleva registros contables y el resto cuaderno de cuentas.

El $83 \%$ ejecutan sus actividades de producción en un taller y el resto en el domicilio, el $90 \%$ de los negocios están en el área urbana y un $10 \%$ en el área rural, el $93 \%$ tienen más de 5 años de antigüedad, el $50 \%$ produce calzados de línea casual, además de zapatos formales, industrial y botas.

¿En qué tipo de local ejecuta sus actividades de producción?

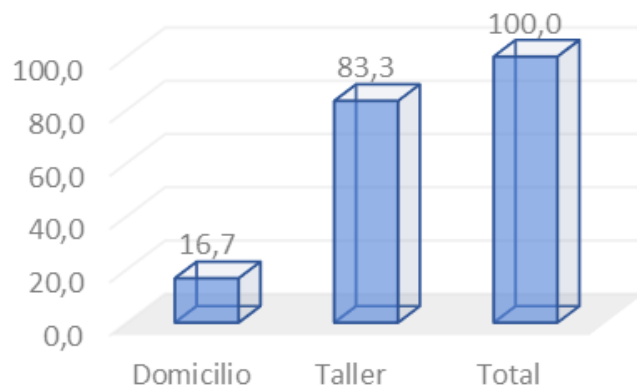

¿En qué zona está localizado su establecimiento?

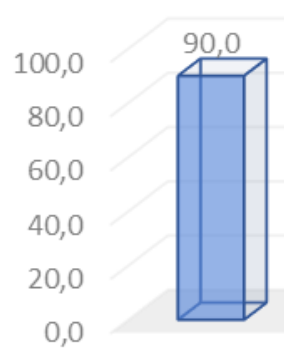

Urbana

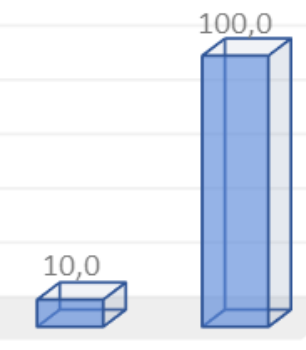

Rural

Total

Resulta importante la planificación de la producción, ya que estima la capacidad productiva de los recursos disponibles teniendo en cuenta la disponibilidad de recursos 
y el conocimiento de la demanda, existen métodos que mejoran la exactitud de los elementos a planificar, sin embargo, el $63 \%$ de las pequeñas empresas no los utilizan. El $66,7 \%$ de los negocios no realiza investigación de mercado para determinar las necesidades, por lo que no recopila información del mercado, lo que le impide establecer las diferentes políticas, objetivos, planes y estrategias más adecuadas a sus intereses.

Los indicadores para medir el desarrollo de la producción son la expresión cuantitativa de su desempeño, esta magnitud, al ser comparada con algún nivel de referencia, puede estar señalando una desviación sobre la cual se toman acciones correctivas o preventivas según el caso y es importante para cuantificar el logro de las metas y la capacidad de lograr el efecto que se desea.

Los resultados señalan que en estos negocios el $50 \%$ no define indicadores para medir el desarrollo de la producción y el $80 \%$ no utiliza ningún método técnico para evaluar la efectividad en la utilización de los recursos.

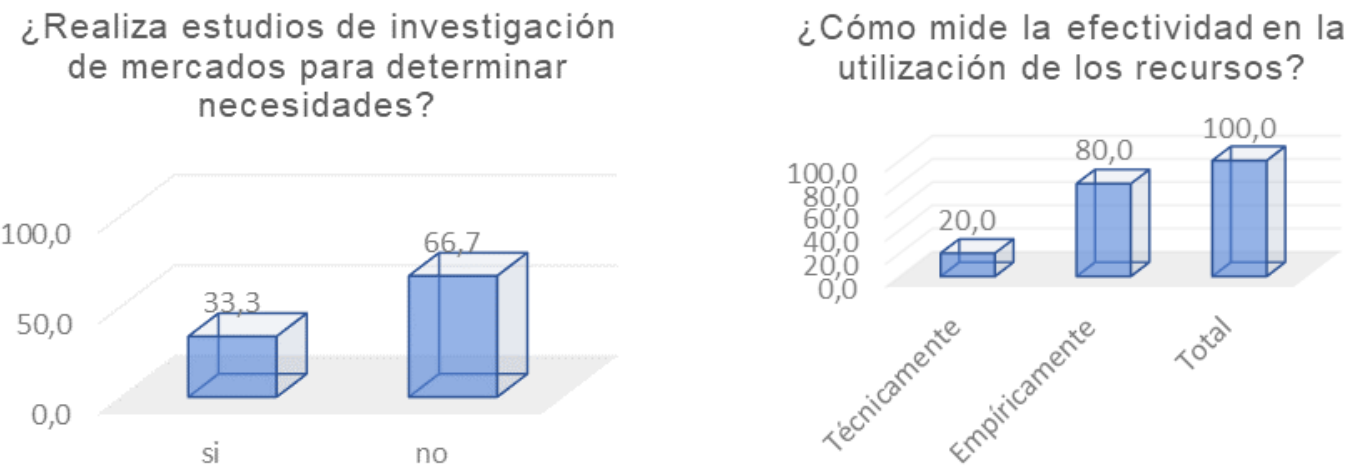

El control de la calidad puede garantizar el cuidado y la mejora continua en la calidad ofrecida y que los productos cumplan con los requisitos mínimos, en función de la satisfacción del cliente, un resultado positivo se identifica que el $60 \%$ de los negocios miden la calidad mediante métodos técnicos.

El $100 \%$ de los negocios tiene identificado sus principales competidores, no obstante, los clientes consideran la calidad de los productos un poco peor y muy por debajo de la 
competencia, con precios menos aceptables, lo que trae como resultado, que los clientes no recomienden los productos que estos ofertan.

El $70 \%$ de los dueños de los negocios valora de regular a malo el desarrollo tecnológico de su negocio, coincide con la percepción de los trabajadores que el 60 \% evalúa de regular a malo, el apoyo tecnológico para realizar su trabajo.

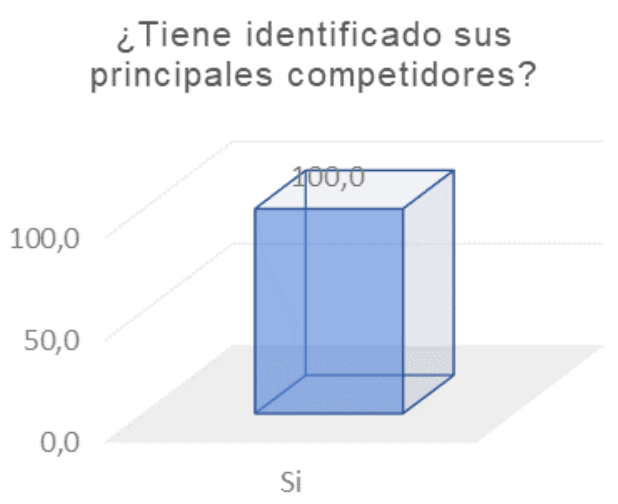

¿Tiene identificado sus

principales competidores?

Si
¿Valore el desarrollo tecnológico de su negocio?

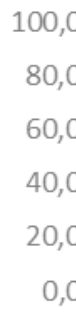

0,0

$100,0 \quad 100,0$

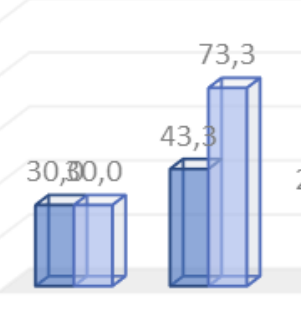

Bueno Regular

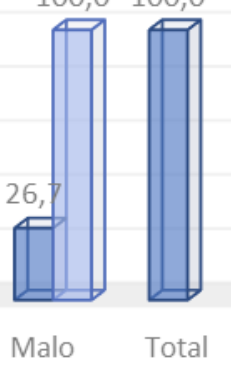

El $66 \%$ está satisfecho con el comportamiento humano individual de sus empleados, se aprecia que se puede aprovechar el hecho de que el 86,7\% de los trabajadores, se sientan involucrados con los éxitos y fracasos de su área de trabajo.

Más del $50 \%$ de los dueños, valora de bueno el trabajo en equipo, sin embargo, el 83 $\%$ de los trabajadores, considera de regular a malo el funcionamiento de la comunicación interna en su área de trabajo.

Con respecto a la capacidad de la organización de convertir las habilidades y recursos en bienes, asegurar, administrar, controlar habilidades, recursos escasos y valiosos, el gobierno lo evalúa de positivo teniendo en cuenta que el sector de cuero y calzado de la provincia de Tungurahua y su cadena de valor produce el $80 \%$ de los 31 millones de pares de que fabrica anualmente la industria ecuatoriana. Mueve USD 600 millones y genera alrededor de 100 mil plazas de empleo directo e indirecto.

En la provincia de Tungurahua se encuentran 2.500 de los 5.000 talleres y empresas medianas, grandes y pequeñas del país, dedicadas a la producción de calzado, esto 
Revista Arbitrada Interdisciplinaria KOINONIA

Año V. Vol V. N9. Enero - Junio 2020

Hecho el depósito de Ley: FA2016000010

ISSN: 2542-3088

FUNDACIÓN KOINONIA (F.K). Santa Ana de Coro. Venezuela.

Juan Carlos Erazo-Álvarez; Cecilia Ivonne Narváez-Zurita

genera miles de plazas de trabajo y su aporte a la economía local y regional es de vital importancia.

En la provincia de Tungurahua se producen 12 de los 14 millones de pares de zapatos anuales y que abastecen el mercado local e internacional. A continuación, en la figura 1 se presentan los principales hallazgos respecto a la medición del capital intelectual en la industria del sector de cuero y calzado de la Provincia de Tungurahua - Ecuador.

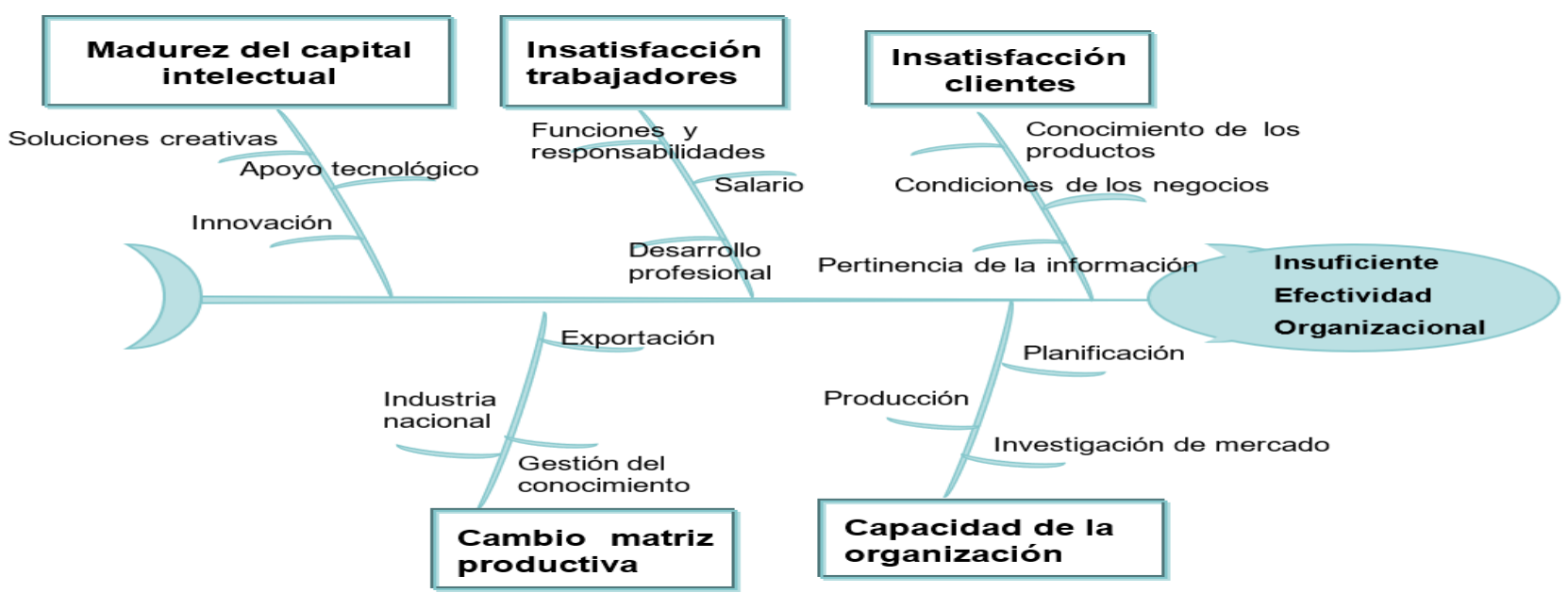

Figura 1. Diagrama de Ishikawa con los resultados de la medición del capital intelectual. Fuente:Elaboración propia, a partir de los resultados del diagnóstico

\section{CONCLUSIONES}

Las causas fundamentales de la insuficiente efectividad se relacionan con la insatisfacción de los clientes, de los trabajadores, con la poca madurez del capital intelectual, con la incapacidad de la organización para asegurar, administrar, controlar habilidades recursos escasos y valiosos y con la no consolidación aun del cambio matriz productiva.

En la insatisfacción de los clientes influye el poco conocimiento de los productos, las malas condiciones de los negocios para brindar el servicio, la poca pertinencia de la información que brinda el negocio sobre sus productos, la desventaja con respecto a la 
Revista Arbitrada Interdisciplinaria KOINONIA

Año V. Vol V. N9. Enero - Junio 2020

Hecho el depósito de Ley: FA2016000010

ISSN: 2542-3088

FUNDACIÓN KOINONIA (F.K). Santa Ana de Coro. Venezuela.

Juan Carlos Erazo-Álvarez; Cecilia Ivonne Narváez-Zurita

competencia en calidad y precio, el mal trato a los clientes y la insuficiente profesionalidad.

En la insatisfacción de los trabajadores, se advierten elementos como laindefinición de funciones y responsabilidades, el desconocimiento de los riesgos y las medidas de prevención relacionadas con su puesto de trabajo, la insatisfacción con las condiciones salariales y con las oportunidades para su desarrollo profesional.

La medición de la madurez del capital intelectual identifica cargas de trabajo mal repartidas, insuficiente apoyo tecnológico para realizar el trabajo, imposibilidadpara proponer mejoras en el trabajo, la insuficiente contribución de los empleados a encontrar soluciones creativas, la poca voluntad de los directivos de innovar para mejorar la calidad de los productos y el mal funcionamiento de la comunicación interna en su área de trabajo.

En la capacidad de la organización para asegurar, administrar, controlar habilidades recursos escasos y valiosos, se aprecia que no se utilizan métodos de planificación, no se realiza investigación de mercado para determinar las necesidades, no se definen indicadores para medir el desarrollo de la producción y no se utilizan métodos para evaluar la efectividad en la utilización de los recursos.

No se consolida aun el cambio de la matriz productiva en estos negocios ya que no hay capacidad para la exportación y es insuficiente investigación e innovación en ciencia y tecnología y gestión del conocimiento.

Teniendo en cuenta que la industria de cuero y calzado en el Ecuador está considerada como una de las actividades dinamizadoras de la economía, el diagnóstico de medición realizado permite concluir que resulta fundamental diseñar una metodología para la gestión del capital intelectual que contribuya al incremento de la efectividad organizacional.

\section{FINANCIAMIENTO}

No monetario 
Revista Arbitrada Interdisciplinaria KOINONIA

Año V. Vol V. N9. Enero - Junio 2020

Hecho el depósito de Ley: FA2016000010

ISSN: 2542-3088

FUNDACIÓN KOINONIA (F.K). Santa Ana de Coro. Venezuela.

Juan Carlos Erazo-Álvarez; Cecilia Ivonne Narváez-Zurita

\section{AGRADECIMIENTO}

A los productores de cuero y calzado de la provincia de Tungurahua por estimular y apoyar esta investigación.

\section{REFERENCIAS CONSULTADAS}

Alama, E., Martin de Castro, G., \& López, P. (2006). Capital intelectual. Una propuesta para clasificarlo y medirlo. [Intellectual capital. A proposal to classify it and measure it]. Revista latinoamericana de administración, 1-16.

Azuara, R. (21 de Marzo de 2018). Organización y estructuras organizacionales. Las organizaciones y la efectividad organizacional. [Organization and organizational structures. Organizations and organizational effectiveness]. Obtenido de Master universitario en Dirección de Empresas online. Calameo: http://es.calameo.com/books/002318012b7dc5f2a0a87

Bernal Ávila, E. M., Erazo Álvarez, J. C., \& Narváez Zurita, C. I. (14 de Septiembre de 2019). Estructuras organizativas favorables a la Eficiencia Empresarial. [Organizational structures favorable to Business Efficiency]. Revista Arbitrada Interdisciplinaria Koinonía, 4(1 (4)), 24. http://dx.doi.org/10.35381/r.k.v4i1.370

Borras, F., \& Campos, L. (2013). Análisis de la gestión y la medición del capital intelectual. [Analysis of the management and measurement of intellectual capital]. Cofinhabana, 36-41.

Castellanos, G., \& Millán, J. (2010). Evolución de la medición y gestión del capital intelectual en una empresa del sector real en Colombia. [Evolution of the measurement and management of intellectual capital in a real sector company in Colombia]. Cali: Universidad Icesi.

Cervera, F. (2011). La percepción de la efectividad organizacional. [The perception of organizational effectiveness]. Querétaro: Universidad Autónoma de Querétaro.

Delgado, M., Martin de Castro, G., Navas, J., \& Cruz, J. (2013). Capital social, capital intelectual e innovación de producto. Evidencia empírica en sectores manufactureros intensivos en tecnología. [Social capital, intellectual capital and product innovation. Empirical evidence in technology-intensive manufacturing sectors]. Innovar, 93-110.

Edvinsson, L. (2007). El capital Intelectual. [Intellectual capital]. Barcelona: Ediciones Gestión 2000. 
Revista Arbitrada Interdisciplinaria KOINONIA

Año V. Vol V. N9. Enero - Junio 2020

Hecho el depósito de Ley: FA2016000010

ISSN: 2542-3088

FUNDACIÓN KOINONIA (F.K). Santa Ana de Coro. Venezuela.

Juan Carlos Erazo-Álvarez; Cecilia Ivonne Narváez-Zurita

Erazo Álvarez, J. C. (2018). El capital intelectual en el sector de cuero y calzado de la Provincia de Tungurahua. [Intellectual capital in the leather and footwear sector of the Tungurahua Province]. REVISTA KILLKANA SOCIALES, 2(3). https://doi.org/10.26871/killkana social.v2i3.333

Ficco, C., Analía, G., \& Sader, G. (2013). Aspectos clave en la revelación del capital intelectual en empresas cotizantes del mercado de capitales argentino. [Key aspects in the disclosure of intellectual capital in listed companies in the Argentine capital market]. Visión Contable, 330-362.

Guerrero, S. (2013). Efectividad Organizacional. [Organizational effectiveness]. Bogota: Universidad Militar Nueva Granada.

Harms, M., Avila, A., Rados, G., \& Rodríguez, C. (2015). Capital intelectual en la gestión pública: caso del método intellectus. [Intellectual capital in public management: case of the intellectus method]. Navus, 103-112.

Kaplan, R., \& Norton, D. (2000). El cuadro de mando integral. [The balanced scorecard]. Madrid: Ediciones 2000.

Kohli, A., \& Jaworski, B. (1990). Marketing Orientation: The Construct, Research Proposition, and Managerial Implications. [Orientación de marketing: la construcción, la propuesta de investigación y las implicaciones gerenciales. Journal of Marketing, 1-18.

Martin de Castro, G., Alama, E., Navas, J., \& López, P. (2009). El papel del capital intelectual en la innovación tecnológica. Un aplicación a las empresas de servicios profesionales. [The role of intellectual capital in technological innovation. An application to professional service companies]. dernos de Economía y Dirección de la Empresa, 083-110.

Monagas, M. (2012). El Capital Intelectual en las empresas hoteleras en Cuba. Procedimiento para su medición. [Intellectual Capital in hotel companies in Cuba. Procedure for its measurement]. Ingeniería Industrial. La Habana: Universidad tecnólogica de La Habana.

Montejano, S., \& Citlalli, G. (2013). Metodología de medición de impacto del capital intelectual en la innovación en empresas: una perspectiva de México. [Methodology for measuring the impact of intellectual capital on business innovation: a perspective from Mexico]. TEACS, 39-50.

Montero, Y., Leyva, E., \& Ballester, T. (2016). Metodología para evaluar la efectividad organizacional. [Methodology to evaluate organizational effectiveness]. Caribeña de Ciencias Sociales. https://n9.cl/sxyn 
Revista Arbitrada Interdisciplinaria KOINONIA

Año V. Vol V. No9. Enero - Junio 2020

Hecho el depósito de Ley: FA2016000010

ISSN: 2542-3088

FUNDACIÓN KOINONIA (F.K). Santa Ana de Coro. Venezuela.

Juan Carlos Erazo-Álvarez; Cecilia Ivonne Narváez-Zurita

Morales, A. (2013). Modelo de gestión de capital intelectual para la pequeña y mediana empresa en México. [Intellectual capital management model for small and medium-sized companies in Mexico]. México DF: Universidad Nacional Autónoma de México.

Ochoa, M., Prieto, B., \& Santidrian, A. (2013). Indicadores de capital intelectual y su relación con el rendimiento. Un análisis empírico. [Indicators of intellectual capital and its relation to performance. An empirical analysis]. Recherches en Sciences de Gestion, 61-79.

Romero, D., Pascual, F., \& Hernández, A. (2012). Gestión del Capital Intelectual: Un reto para la gerencia de la pequeña y mediana empresa latinoamericana. Formación Gerencial, 1-36. [Intellectual Capital Management: A challenge for the management of small and medium Latin American companies. Management Training, 1-36].

Romero, J., \& Salvador, R. (2005). Gestión del Conocimiento y del Capital Intelectual en una PYME. [Knowledge Management and Intellectual Capital in an SME]. Barcelona: Universidad Politécnica de Catalunya.

Romero, J., \& Salvador, R. (2008). Gestión del Conocimiento y del Capital Intelectual en una PYME del sector textil. Barcelona: Universidad Politécnica de Catalunya. [Knowledge Management and Intellectual Capital in a SME of the textile sector. Barcelona: Polytechnic University of Catalonia].

Sosa, M. (2013). Capital intelectual en mercados en desarrollo. "El caso Paraguay". [Intellectual capital in developing markets. "The Paraguay case"]. Sevilla: Universidad de Sevilla.

Valencia, M. (2005). El capital intelectual: capacidad competitiva empresarial. [Intellectual capital: competitive business capacity]. Entramado 1(1), 40-49.

(C2020 por los autores. Este artículo es de acceso abierto y distribuido según los términos y condiciones de la licencia CreativeCommons Atribución-NoComercial-Compartirlgual 4.0 Internacional (CC BY-NC-SA 4.0)

(https://creativecommons.org/licenses/by-nc-sa/4.0/). 